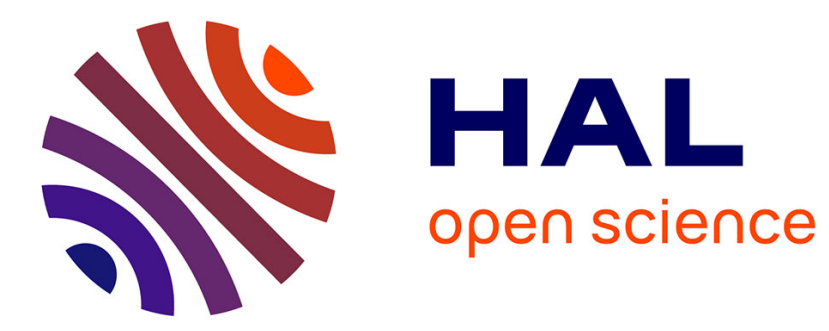

\title{
Diachronic'Explorer: Keep track of your clusters!
}

\author{
Nicolas Dugué, Jean-Charles Lamirel, Pascal Cuxac
}

\section{To cite this version:}

Nicolas Dugué, Jean-Charles Lamirel, Pascal Cuxac. Diachronic'Explorer: Keep track of your clusters!. RCIS, Jun 2017, Grenoble, France. pp.1-2, 10.1109/RCIS.2016.7549367 . hal-01444752

\section{HAL Id: hal-01444752 \\ https://hal.science/hal-01444752}

Submitted on 24 Jan 2017

HAL is a multi-disciplinary open access archive for the deposit and dissemination of scientific research documents, whether they are published or not. The documents may come from teaching and research institutions in France or abroad, or from public or private research centers.
L'archive ouverte pluridisciplinaire HAL, est destinée au dépôt et à la diffusion de documents scientifiques de niveau recherche, publiés ou non, émanant des établissements d'enseignement et de recherche français ou étrangers, des laboratoires publics ou privés.

\section{(c)(1)}

Distributed under a Creative Commons Attribution| 4.0 International License 


\section{Diachronic'Explorer : keep track of your clusters !}

\author{
Nicolas Dugué, Jean-Charles Lamirel \\ LORIA-Synalp \\ Vandoeuvre lès Nancy, France. \\ e-mail : first.last@loria.fr
}

\author{
Pascal Cuxac \\ CNRS-INIST \\ Vandoeuvre lès Nancy, France. \\ e-mail: pascal.cuxac@inist.fr
}

\begin{abstract}
We introduce Diachronic'Explorer, a toolbox to produce and visualize diachronic results, which is based on a new complete theoretic framework for diachrony that we detail. This toolbox, which is dedicated to run diachronic algorithms from clustering results, allows also to explore complex diachronic results at all the granularity levels through a web application.
\end{abstract}

\section{INTRODUCTION}

The ISTEX project compiles and provides access to scientific publications to French research. ISTEX-R project aims to highlight evolution of research topics using the ISTEX database. To that aim, we designed a new theoretical framework to track scientific fields changes and similarities across time : appearing and disappearing topics, splitting and merging topics. This framework is based on diachronic analysis, which aims to compare data or results from two distinct periods. Furthermore, this framework was implemented as a toolbox called Diachronic'Explorer that integrates all diachronic analysis steps and provides efficient visualizations.

In the context of our framework, we basically consider $t p$ time periods, containing documents split into separate sets according to these time periods and described by a set of features $F$ (words or expressions) : $D=\cup_{0 \leq i \leq t p} D_{i}$. For each time period, clustering algorithms detect cluster sets such as $C S=\cup_{0 \leq i \leq t p} C_{i}$. Each set of clusters $C_{i}=\left\{c_{1}, \cdots, c_{n_{i}}\right\}$ is constituted of $n_{i}=\left|C_{i}\right|$ clusters describing data $D_{i}$. Clusters gather similar documents and thus represent topics. Consequently, to monitor topic changes, our framework aims to track cluster changes and similarities across time periods.

The first step of our new framework consists in labeling clusters of every cluster sets. These labels will then be used to track cluster changes and similarities. Our labeling step consists in extracting prevalent features sets $S_{c_{j}}$ for each cluster $c_{j} \in C_{i}$ of all the cluster sets $C_{i} \in C S$ using a feature selection method. We implement the feature maximization method proposed by Lamirel et al. [3] that has proven to be efficient for both supervised and unsupervised learning. The second step processes diachronic analysis between time periods using cluster labels. Basically, using $S_{c_{j}}$, the prevalent features sets of each cluster, we apply a diachronic algorithm based on MVDA, an unsupervised Bayesian process operating between views (here periods) for diachronic mining [4].

\section{FEATURE SELECTION FOR CLUSTER LABELING}

The first step of our framework consists in labeling clusters using a feature selection method. The one we implement is

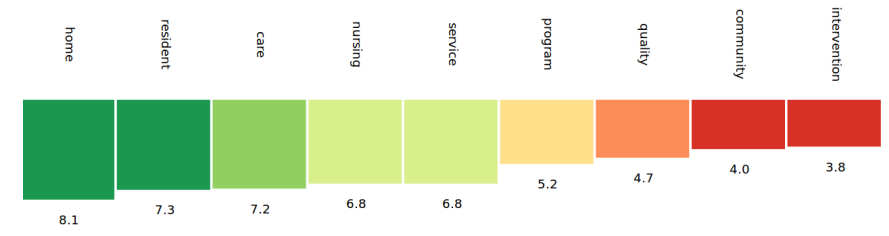

Fig. 1. Cluster labels extracted and visualized with Diachronic'Explorer.

non-parametric, only weakly influenced by feature scaling, and it allows to label clusters with ordered weighted features [3]. We apply it independently to each period. Thus, for each cluster set $C_{i}, 0 \leq i \leq t p$, two ratios are processed over each cluster $c_{j} \in C_{i}, 0 \leq j \leq n_{i}$ to give a weight to features of $F$ for the cluster considered. On the first hand, Feature Predominance (Equation 1) aims to evaluate if a feature $f \in F$ allows to discriminate $c_{j}$ from other clusters of $C_{i}$. On the other hand, Feature Recall (Equation 2) aims to evaluate whether $f$ allows to faithfully describe data in $c_{j}$.

$$
F P_{c_{j}}(f)=\frac{W_{c_{j}}^{f}}{W_{c}} \quad \text { (1) } \quad F R_{c_{j}}(f)=\frac{W_{c_{j}}^{f}}{W_{D_{i}}^{f}}
$$

with $W_{c_{j}}^{f}=\sum_{d \in c_{j}} W_{d}^{f}$ the weighted sum of $f$ across cluster $c_{j}, W_{D_{i}}^{f}=\sum_{d \in D_{i}} W_{d}^{f}$ the weighted sum of $f$ across the data of the time period considered, $W_{c_{j}}=\sum_{f^{\prime} \in F} W_{c_{j}}^{f}$ the weighted sum of all features across cluster $c_{j}$. The Feature $F$-Measure $F F_{c_{j}}(f)$ is the harmonic mean of these two values.

The Feature F-Measure is thus used to select features that are discriminant and representative for each cluster $c_{j}$ using Equation 3.

$$
S_{c_{j}}=\left\{f \in F \mid F F_{c_{j}}(f)>\overline{F F_{C_{i}}(f)}, F F_{c_{j}}(f)>\overline{F F_{D_{i}}}\right\}
$$

with $\overline{F F(f)}$ the mean F-Measure value of $f$ across clusters of $C_{i}$ where $f$ is non-null, and $\overline{F F_{D_{i}}}$ the mean F-Measure across all features and all data of the considered period. Figure 1 shows how features selected for a cluster with Diachronic'Explorer are used as labels to describe the cluster in the visualization module.

\section{DiACHRONY WITH MVDA}

Once the representative labels of each cluster extracted, our second step consists in applying MVDA diachronic analysis between each time periods pairs. MVDA was firstly experimented as a fully unsupervised approach by Lamirel [4], 


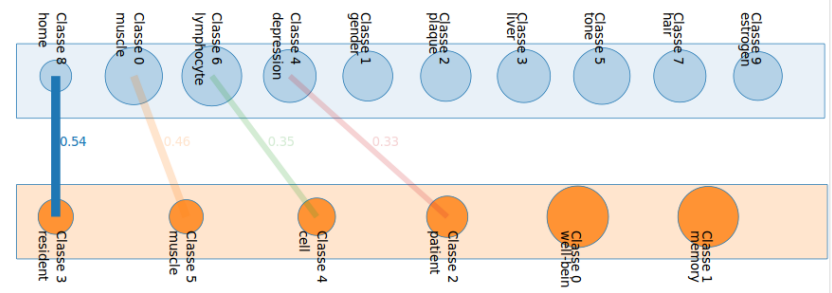

Matching information

Matching type : Bidirectional

Label home is common to Classe8 (8.083723) and Classe3 (4.210675)

Label resident is common to Classe8 (7.333534) and Classe3 (5.587822

Label nursing is common to Classe8 (6.828802) and Classe 3 (5.058351)

Label quality is common to Classe8 (4.677062) and Classe3 (3.278548)

Fig. 2. Exploring matchs between two sets of clusters using $\mathrm{Di}$ achronic'Explorer. The matching kernel of Classe 8 and Classe3 is displayed in the yellow box while the match strength between these clusters is represented with the thickness of the link between them.

but here, we use cluster labels instead of indexer keywords. This process is theoretically based on Bayesian reasoning [4] whose operating mode can be summarized as this in our context : the diachronic analysis is processed by computing matching probabilities between clusters of distinct sets (thus time periods) using the selected labels they share or not. The matching probability of a cluster $s$ from a set $C_{s r c}$ and a cluster $t$ from a set $C_{t g t}$ is computed as shown in Equation 4.

$$
P(t \mid s)=\frac{\sum_{f \in S_{s} \cap S_{t}} F F_{t}(f)}{\sum_{f \in S_{t}} F F_{t}(f)}
$$

The matching probability between $t$ and $s$ is processed symmetrically. Finally, an asymmetric match from $s$ to $t$ is detected if the matching probability $P(t \mid s)$ is greater than the average matching probability of $s$ with the clusters of $C_{t g t}$. A symmetric match exists if both asymmetric match from $s$ to $t$, and from $t$ to $s$ are detected. In such a case, the set of features describing this match is called the matching kernel (Figure 2). These different kind of match detected by Diachronic'Explorer allow us to track and describe changes and similarities between cluster sets of distinct periods.

\section{EXPERIMENTAL RESULTS AND VISUALIZATIONS}

Experiments and visualizations shown in this papers were conducted on a corpus constituted of bibliographic data from the ISTEX project [6]. The ISTEX database is queried to extract papers related to research in medical care between years 1996 and 2010. This results in a dataset of 9779 papers. Because the goal is to observe evolution of this field across time, a community detection algorithm [5] exploiting the relationship between documents indexes and the publication years is used to extract meta-periods. Obtained meta-periods are 1996-2000, 2000-2005, 2006-2010 [1]. GNG clustering [2] is launched several times on the data of each meta-period to extract an optimal number of clusters with the help of adhoc quality indexes [4]. Then, using Diachronic' Explorer $^{1}$, we

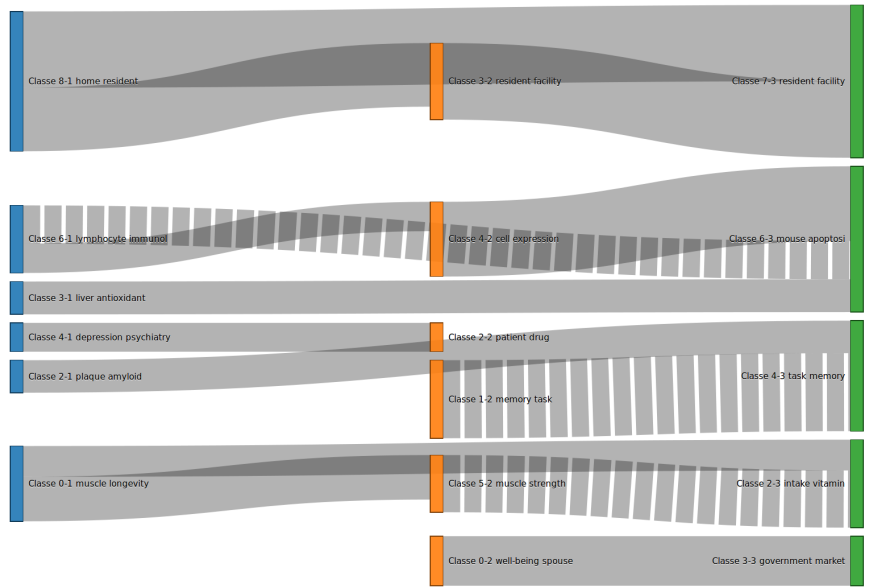

Fig. 3. Exploring cluster labels of a cluster set using Diachronic'Explorer. Each column/color describes a period where rectangles stands for labels clusters. Grey flows between the colored rectangles represent different kind of matchs (asymmetric/symmetric) and their strength.

produce and then visually explore the diachronic results with powerful dynamic visualizations using advanced Javascript ${ }^{2}$. Visualization tool of Diachronic'Explorer makes possible to navigate easily from one granularity level to another : cluster and their labels (Figure 1), match between two periods (Figure 2), overall dataset evolution (Figure 3).

\section{CONClusion}

Integrating a new framework using powerful labeling method and unsupervised Bayesian reasoning among clusters sets, Diachronic'Explorer allows to produce diachronic results and provides a web application to visualize these results, navigating easily from one granularity level to another.

Acknowledgments. ISTEX receives assistance from the French state managed by the National Research Agency under the program Future Investments bearing the reference ANR10-IDEX-0004-12.

\section{REFERENCES}

[1] Cuxac, P. and Lamirel, J. C. Analysis of evolutions and interactions between science fields: the cooperation between feature selection and graph representation. 14th COLLNET Meeting (2013)

[2] Fritzke, B. A growing neural gas network learns topologies. Advances in Neural Information Processing Systems 7, 625-632 (1995)

[3] Lamirel, J.-C., Cuxac P., Chivukula A.S., Hajlaoui K.: Optimizing text classification through efficient feature selection based on quality metric. Journal of Intelligent Information Systems, 2013, 1-18 (2014)

[4] Lamirel, J.-C. : A new approach for automatizing the analysis of research topics dynamics: application to optoelectronics research. Scientometrics 93(1), 151-166 (2012)

[5] Pons, P. and Latapy, M.: Computing Communities in Large Networks Using Random Walks. Journal of Graph Algorithms and Applications, 10(2): 191-218 (2006)

[6] Dugué, N. and Tebbakh, A. and Cuxac, P. and Lamirel, J.-C.: Feature selection and complex networks methods for an analysis of collaboration evolution in science: an application to the ISTEX digital library. 5th International Symposium ISKO-MAGHREB (2015)

\footnotetext{
${ }^{1}$ Demo - Choose "2tiers1tiers" experiment - http://107.170.67.36:3000/

Git - https://github.com/nicolasdugue/istex-demonstrateur

${ }^{2} \mathrm{https}: / / \mathrm{d} 3 \mathrm{js}$. org/
} 